\title{
A Strange Case of Ovarian Cancer's Recurrence: Is the Follow up Safe and Effective?
}

\author{
Macina S*, Barzacchi C, Vita M and Razzetta F \\ Sestri Levante Hospital Asl 4 Chiavarese, University of Study of Genova, Italy
}

*Corresponding author: Macina S, Sestri Levante Hospital Asl 4 Chiavarese, University of Study of Genova, Italy, Tel: 00393484282344, E-mail: simona.macina@gmail.com

Citation: Macina S, Barzacchi C, Vita M, Razzetta F (2018) A Strange Case of Ovarian Cancer's Recurrence: Is the Follow up Safe and Effective? SAJ Case Rep 5: 201

Article history: Received: 14 March 2018, Accepted: 1 May 2018, Published: 3 May 2018

\begin{abstract}
We present a case of a woman, with a previous diagnosis of ovarian cancer. She underwent an istero-annessiectomy and bilateral iliac lymphadenectomy and adjuvant complete cycle of chemotherapy. During next follow up, in the CT scan there was the evidence of a big neoplasm close to the left colon. All the radiological, cytological and biochemical exams were negative for recurrence. During surgical operation for a big ventral incisional hernia, we decided to take a sample of the neoplasm, which was positive for recurrence of the primary ovarian cancer. She underwent left hemicolectomy.
\end{abstract}

Keywords: Ovarian Cancer; Recurrence; Left Hemicolectomy

\section{Introduction}

Ovarian cancer includes a variety of several histopathological entities; the most frequent one is the epithelial cancer (about 90\%) even if other subtypes may occur; the diagnosis and the treatment depend on the specific tumor [1,2]. It is the fifth most common cancer in US and the number of new diagnosis is around 22,000 in 2016, with the prevalence in the sixth and seventh decades [3].

The risk factors are: nulliparity, older age at the first pregnancy, post-menopausal hormone therapy, pelvic inflammatory disease, and positive family history (including linking to BRCA1 and BRCA2 genotypes, affection by Lynch syndrome) [4-7].

Symptoms suggestive of ovarian cancer include: bloating, pelvic or abdominal pain, difficulty eating or feeling full quickly, urinary problems like urgency or frequency [8]. There are no screening tests available, but woman with high risk should get deeper evaluation [9].

Primary treatment consists of appropriate surgical staging, followed very often by a systemic chemotherapy [10]. After initial treatment, the patient should undergo clinical re-evaluation and follow-up. Ovarian cancer has a property of persistence after primary surgery and chemotherapy even in early stages, so it is very important to have an accurate diagnosis of recurrence in order to follow up these patients properly. Recurrent disease may be identify clinically, biochemically and with imaging (ultrasound, chest and abdomen CT scan and FDG-PET scan if clinically necessary) [11].

\section{Case Report}

We present a case of 57 years old woman who had in 2013 a bilateral ovarian adenocarcinoma. Her medical history was positive for obesity, diabetes mellitus and laparoscopic cholecystectomy. She underwent an explorative laparotomy. The gynecologist decided to perform a bilateral salpingo-ovaro annessiectomy, an enblock Douglasectomy, a bilateral pelvic lymphadenectomy, an appendicectomy and a inframesocolic omentectomy. The definitive histological response confirmed the preoperative diagnosis: a bilateral endometrioid adenocarcinoma with omental metastasis without the involvement of lymph nodes (pT3c/G2/pN0; Figo IIIC stage). After one month, she started the adjuvant chemotherapy with Carbo Auc 5 Taxolo and she finished all the cycles in May 2013 with a complete remission.

In the next years, she underwent a correct follow up with the dosage of biochemical markers, CT scan and PET scan, all negative. In September 2016 during a PET scan, it was visible a big hypo dense photopenic area (around $12 \times 13 \mathrm{~cm}$ ), localized in front 
of and under the left kidney, without 18F-FDG enhancing. The patient did not complain any symptoms related. She had also a CT scan, which confirmed the presence of a big anaecogen area with cystic aspect with irregular tick wall in the left abdomen. The meantime, all biochemical markers (CEA, CA 19.9, CA 125) were negative. She underwent also an ultrasound guided ago biopsy of the lesion and the cytological exam was suitable to a cystic formation, negative for cancer cells.

The Oncologist asked for a surgical opinion because of the presence of a big medial incisional hernia. During the first counseling with the Oncologists and Radiologists, the final decision was to repair the abdominal hernia with a preperitoneal mesh. In March 2017 the patient underwent the procedure, but in the operation room the surgical equipe decided to open the peritoneum, get inside the abdominal cavity and reach the neoplasm (Figure 1). It was brown colored, with tick wall. The first step was the aspiration of the liquid part of the cyst (about $200 \mathrm{cc}$ ), that appeared to be brown; it was sent to cytological and biochemical exam. The macroscopic aspect it was suggestive for pancreatic pseudocyst. At the opening of the the wall, attached to the left colon, there were some vegetations, sent to histological exam. The operation ended with no mesh repair of the abdominal wall. The cytological exam was negative for malignant cells. The histological response of the inside-cyst vegetations was indicative for a secondary localization of the ovarian adenocarcinoma endometroid type. The pathologists underlined that the review of the previous histological samples of the 2013 evidenced a morphological pattern completely suitable with a metastatic localization of the endometroid carcinoma with ovarian primitivity. With this histological response, the multidisciplinary team decided to perform an open left hemicolectomy. The patient was discharged in the sixth post-surgical day, with no complications. The Oncologists decided with the patient to start a new cycle of chemotherapy.

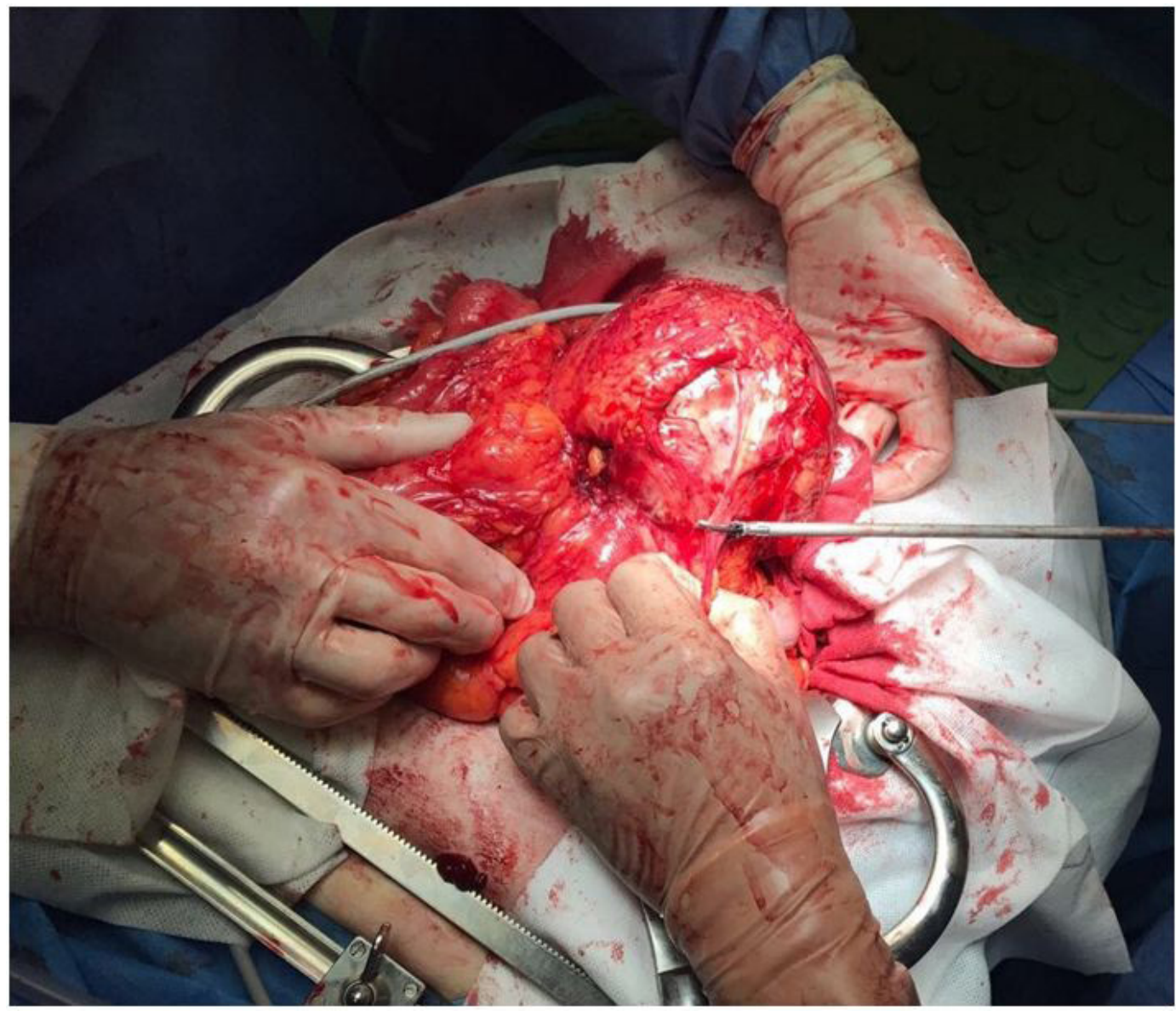

Figure 1: Exegetical surgical procedure of the abdominal mass

\section{Discussion}

Ovarian cancer has been recently divided into two groups: type I involve low grade serous carcinoma of low grade, low grade of endometrioid carcinoma, clear cells carcinoma, mucinous carcinoma and Brenner tumors. These neoplasms have a good prognosis, rarely present mutations of p53 gene and are genetically stable; type II, represent the $75 \%$ of cases, include high-grade serous carcinoma, high-grade endormetrioid carcinoma, not differentiated carcinoma and carcinosarcoma. They have aggressive biological behavior, they are diagnosed in ad advanced stage, and they have p53 gene mutation and in the $80 \%$ of cases are genetically unstable [12].

Endometrioid carcinomas account for $8-15 \%$ of all ovarian carcinomas and it is the second commonest malignant ovarian neoplasm. These tumors appear similar to other epithelial lesions, with variable cystic and solid components. Histologically, an endometrioid carcinoma is characterized by the appearance of tubular glands [13]. 
Radiographic findings are often non-specific and include a large, complex cystic mass with solid components. There may be associated endometrial thickening, evidence of endometriosis or a contralateral mass [14].

Ovarian cancer has the propency to recurrence even after primary surgery and chemotherapy. The most frequent pattern of spread is the Trans colonic route, even if the intraperitoneal, lymphatic and haematogenous ones may occur [15].

Literature data indicate recurrence in $25 \%$ of early stage diagnosed cases and $80 \%$ of advanced ones [16]. So it is essential an accurate diagnosis of recurrence with a proper follow up methods.

The serum Ca 125 is commonly used, but it is not accurate because the normal serum level doesn't exclude the presence of the disease and doesn't give any information on the site. A negative Ca125 has been seen in patients with residual tumor after surgical procedure.

The imaging such as ultrasound, CT scan and MRI has a role in the characterization of the disease recurrence, even if it's not so certain for determining the disease status.

Positron emission tomography with FDG might represent a valid option is staging and following up a wide variety of neoplasm, even if it hasn't been definitively established [17]. Evaluated the use of 18FDG-PET as a prior test to plan surgical second look and it was positive just in the half of the analyzed patients.

So the best follow up technique is still controversial and there are no unique guidelines to respect.

In most part of surgical centers a second look surgery might be acceptable, because it might be considered the best method to define the disease status; even if it's not helpful with not visible metastasis and long distance lesions.

\section{References}

1. Kurman RJ, Carcangiu ML, Herrington CS, Young RH (2014) WHO Classification of tumors of female reproductive organs (4 $4^{\text {th }}$ edn). WHO/IARC 6 Lyon: IARC Publication.

2. Jevolac D, Armstrong DK (2011) Recent progression in diagnosis and treatment of ovarian cancer. CA Cancer J Clin 61: 183-203.

3. Siegel RL, Miller KD, Jamal A (2016) Cancer Statistics. CA Cancer J Clin 66: 7-30.

4. Mørch LS, Løkkegaard E, Andreasen AH, Kjaer SK, Lidegaard O (2012) Hormone therapy and different ovarian cancer: a national cohort of study. AM J Epidemiol 175: 1234-42.

5. Lin HW, Tu YY, Lin SY, Su WJ, Lin WL, et al. (2011) Risk of ovarian cancerin women with pelvic inflammatory disease: a population based study. Lancet Oncol 12: 900-4.

6. Nakonechny QB, Gilks GB (2016) Ovarian Cancer in Hereditary Cancer Suscettibility Syndromes. Surg Pathol Clin 9: 189-99.

7. Liu G, Yang D, Sun Y, Shmulevich I, Xue F, et al. (2012) Different clinical impact of BRCA1 and BRCA2 mutations in serous ovarian cancer. Pharmacogenomics 13: 1523-35.

8. Goff BA, Mandel LS, Drescher CW, Urban N, Gough S, et al. (2007) Development of an ovarian cancer symptom index: possibilities for an earlier detection. Cancer 109: 221-7.

9. Clarke-Pearson DL (2009) Clinical practice. Screening of ovarian cancer. N Eng J Med 361: 170-7.

10. NCCN Guidelines version 1 (2017) Ovarian cancer.

11. Picchio M, Sironi S, Messa C, Mangili G, Landoni C, et al. (2003) Advanced Ovarian Carcinoma: usefulness of [18F] FDG-PET in combination with CT for lesion detection after primary treatment. Q J Nucl Med 47: 77-84.

12. AIOM Guidelines (2017) ovarian carcinoma.

13. Wagner BJ, Buck JL, Seidman JD, McCabe KM (1994) From the archives of the AFIP. Ovarian epithelial neoplasms: radiologic-pathologic correlation. Radiographics 14: 1351-74.

14. Kinoshita T, Ishii K, Naganuma H, Higashiiwai H (2000) MR findings of ovarian tumors with cystic components. Br J Radiol 73: 333-9.

15. Zighelboim I, Broaddus R, Ramirez PT (2004) Atypical Sigmoid metastasis from a high grade mixed adenocarcinoma of the ovary. Gynecol Oncol 94: 850-3.

16. Trimbos JB, Parmar M, Vergote I, Guthrie D, Bolis G, et al. (2003) International Collaborative Ovarian Neoplasm trial 1 and Adjuvant Chemotherapy In Ovarian Neoplasm trial: two parallel randomized phase III trials of adjuvant chemotherapy in patients with early-stage ovarian carcinoma. J Natl Cancer Inst 95: 105-12.

17.Karlan BY, Hawkins R, Hoh C, Lee M, Tse N, et al. (1993) Glaspy J Whole-body positron emission tomography with 2-[18F]-fluoro-2-deoxy-D-glucose can detect recurrent ovarian carcinoma. Gynecol Oncol 51: 175-81. 